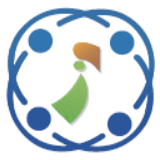

\title{
Optimized Energy Efficient Hybrid Routing Protocol (EE-HRP) for Minimum Battery Power Consumption in MANET
}

\author{
Sushma Ghode $^{1 *} \quad$ Kishor Bhoyar $^{2}$ \\ ${ }^{I}$ Department of Computer Technology, Yeshwantrao Chavan College of Engineering, Nagpur, India \\ ${ }^{2}$ Department of Information Technology, Yeshwantrao Chavan College of Engineering, Nagpur, India \\ * Corresponding author's Email: sushdg@ gmail.com
}

\begin{abstract}
Mobile Ad-hoc networks (MANET) are being installed in a wide range of applications like military scenarios, rescue operations, data and device networks etc. Many of these networks uses battery operated nodes. A major requirement of these networks is to wisely utilize the battery power of nodes, so that the nodes can actively participate in process of communication for maximum time span without partitioning the network. This will result into maximum network lifetime which is again main goal in ad hoc routing algorithms. To achieve this we proposed an optimized Energy Efficient Hybrid Routing Protocol (EE-HRP) which uses routing strategy that seeks to find the best balance between minimizing the power consumption and evenly using all nodes within the network to avoid early exhaustion of individual node. In EE-HRP we use multiple threshold values for remaining battery power of nodes and assign different role of each node depending on remaining battery power of the node using our Node Energy Monitoring Algorithm (NEMA). Here we also make use of our previously designed algorithm Zone Head Selection Algorithm (ZHSA) which is used to select maximum energy node as Zone Head (ZH). By doing so we prevent nodes from early battery exhaustion which results to increase network lifetime to some extent. The key factor governing the performance of optimized EE-HRP are theoretically analyzed and evaluated. The simulation results are compared with some MANET routing protocols in different network scales, taking into consideration the power consumption. The theoretical analysis and simulation results shows that our proposed optimized EE-HRP reduces total power consumption, reduces end-to-end delay, increases packet delivery ratio and achieves maximum network lifetime.
\end{abstract}

Keywords: MANET, Ad hoc network, Hybrid routing protocol, Network lifetime, NEMA, ZHSA.

\section{Introduction}

Mobile Ad-hoc network (MANET) is an infrastructure-less network which is collection of autonomous nodes that moves with some speed and operate without any central administration. These nodes are battery powered which is the only source of energy. MANET is widely used for many commercial as well as domestic purposes like rescue operations, data and device networks, battle fields etc In many of these applications it is not possible to deploy network infrastructure and hence the network is rely only on nodes battery power. In such type of applications it is important to manage communication path between the nodes for maximum period of time so that the nodes can actively participate in communication process [1]. Thus, MANET receives significant research attention and many routing algorithms are developed. The most popular method which is used to distinguish mobile ad hoc network routing protocols is how routing information is acquired, stored and maintained by mobile nodes, by using this MANET routing protocols can be divided into proactive routing, reactive routing and hybrid routing [2].

Further, the Energy efficient routing protocols for MANET are broadly categorized based on when energy optimization is performed [3]. The mobile node consumes its battery energy in performing operations either in active or idle communication 


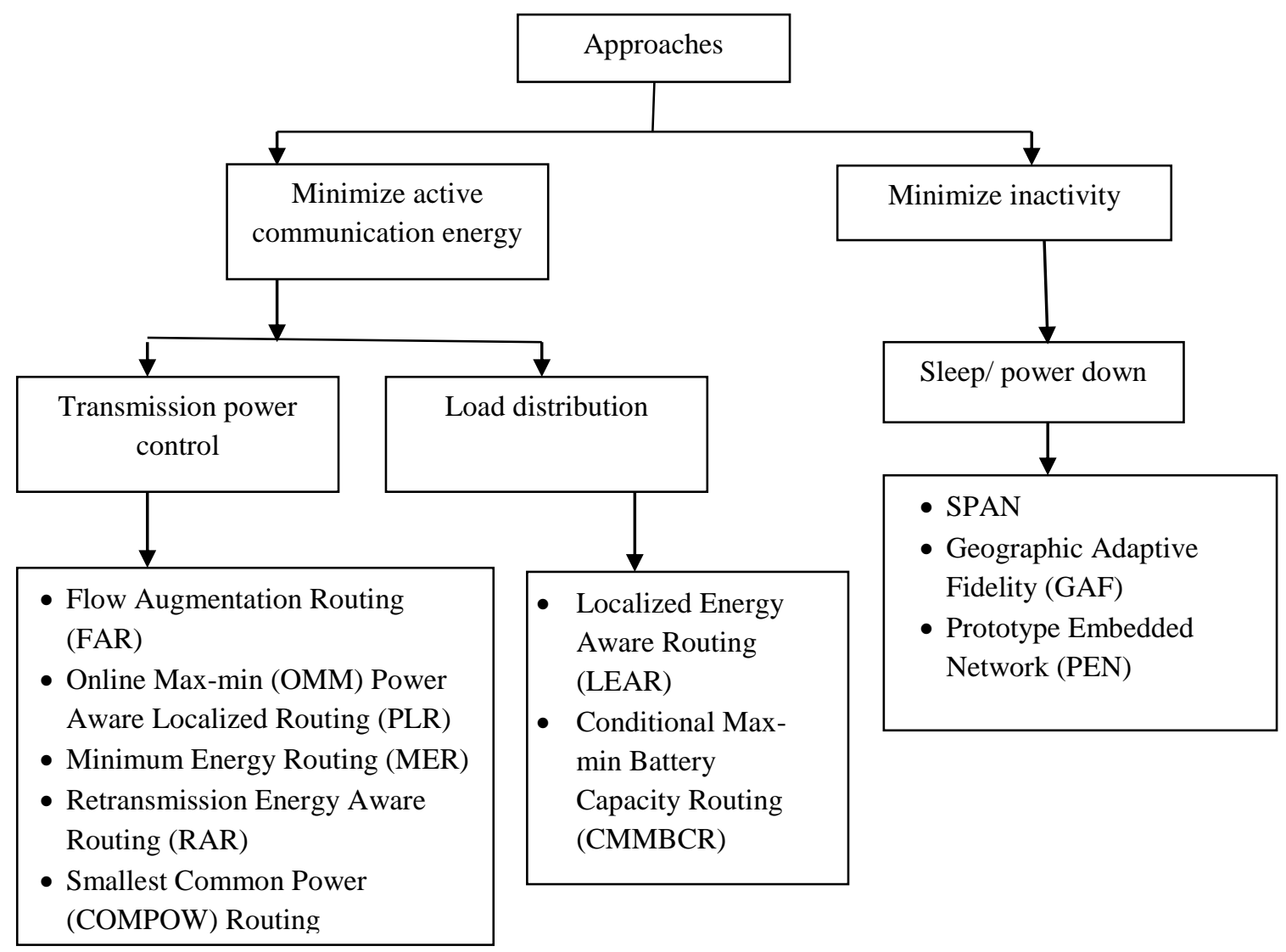

Figure. 1 Different approaches of energy efficient routing algorithm

mode. The Energy efficient routing protocols either minimize active communication energy [4] which is used to send and receive data packets or inactive communication energy [5] which is used to perform internal computational operations. Transmission power control and load distribution approach is comes under prior category while sleep/power down approach comes into later [6]. In sleep/power down approach a proper wake up schedule has to be set to make the nodes to go in periodic sleep mode [7]. The details of these categories are shown in Fig. 1 above.

Network lifetime is a time at which first node in the network is runs out of energy to participate in communication process. Thus in such an environment we can take following measures to extend network lifetime [8]:

1. Monitor the power consumption of individual nodes so that nodes will not die due to energy exhaustion.

2. Distributing the workload of nodes throughout the network to avoid consumption of battery energy of single node.

3. Avoid minimum energy path for communication.
To implement all these measures in one protocols really a critical issue. Many researchers proposed various techniques to accommodate above mention properties in their work. Some uses cross layer, multicast designs by combining network layer and MAC layer to reduce power consumption and improves network lifetime $[9,10]$. We use mechanism of Power aware Localized Routing (PLR) which comes under transmission power control approach, Localized Energy Aware Routing (LEAR) Protocol, which comes under load distribution approach and SPAN protocol which comes under sleep/power down approach of energy efficient routing for MANET for making a hybrid routing protocol which we called as Energy Efficient Hybrid Routing Protocol (EE-HRP). In PLR source node knows link cost from itself to its neighbor and to destination [11]. Based on this source can select next hop through which overall transmission power to the destination is minimum. This selected path may not be the optimal path but as per super-linear theory between transmission power and distance [12] indirect path via intermediate nodes consumes less energy as compare to direct path. In LEAR node determines whether to forward route-request packet or not 
depending on its residual energy [13]. The node forward route-request packet only if its residual energy is above threshold otherwise the packets are dropped. Thus only the nodes having maximum remaining energy is participated in data transmission and nodes with low battery power can conserve their energy [14]. In SPAN the nodes are either identified as coordinator nodes or non-coordinator nodes [15]. The coordinator nodes continuously in idle mode while non-coordinator node can periodically in sleep or awake mode and participated in coordinator election. The coordinator act as base station that buffer data intended for non-coordinator nodes when they are in sleep mode. We use all these features in our proposed EE-HRP protocol to improve its energy efficiency.

The goal of this paper is to design a hybrid routing protocol which uses best of the three routing approaches of energy efficient routing for MNAET which are mentioned herewith viz., transmission control, load distribution and sleep/power down approach. In our proposed protocol, we try to reduce power consumption by using indirect link from source to destination as mentioned in PLR which is one of the protocols in transmission power control approach. Also the relay nodes that act as intermediate nodes to send data from source to destination are selected by using maximum residual energy available in nodes. Thus nodes having less energy are eliminated from route selection as in case with LEAR protocol in load distribution approach. To override network partition caused due to battery power drain of a node, we made minimum residual energy node to go in sleep mode. This may improve network lifetime to some extent. Thus we can use sleep/power down approach monitored by zone head as mentioned in SPAN protocol.

The paper is further organized as follows: in section 2 we describe some related work towards the energy efficient routing in MANET. In section 3 we describe working of our proposed protocol, in section 4 we do performance evaluation of our work using simulation results and compare our EE-HRP with some existing protocols and in section 5 we conclude our work.

\section{Related work}

Many researchers have proposed various methods to improve energy efficiency of routing protocols in MANET. Some of these are mentioned here.

In [16] author modifies DSR algorithm to improve network lifetime. In this the author proposed an efficient DSR (EDSR) which minimizes energy consumption per packet, maximizes network lifetime and minimize maximum node cost. This is done by selecting energy efficient paths. In DSR some relay nodes may act selfish and drop packets for other nodes so that their energy consumption is reduced. The EDSR finds such selfish nodes and deals with them. The drawback of EDSR is to find such selfish node extra overload generated which causes energy consumption. In [17] authors designed an ondemand Power and Mobility Aware Routing (PMAR) protocol. The main objective of PMAR is to select a route that improves the minimum battery power of the node which maximizes network lifetime and reduces total transmission power required to reach destination so that overall energy resources in the network can be utilized efficiently. PMAR also consider mobility of the nodes due to which frequent link breakages occurs in network which affects ongoing data transmission and end-toend delay performance of the MANET. To optimize this problem PMAR adds route lifetime as constraint in the protocol. But all these problems cannot be solved online for large networks hence some heuristic algorithms are designed to solve all these issues in PMAR.

In [18] authors proposed a novel energy consumption model using Residual Energy based Mobile Agent selection scheme (REMA). In this by sharing topology information among the nodes, mobile agent dynamically select appropriate upper layer agents for reliable data transmission. REMA uses multimode Gateway selection process to analyze mobility weightage within the zone group and across different zone groups also congestion free traffic is monitored. REMA retains energy of the node for maximum time to increase battery life and the gateway is selected based on maximum weight which utilizes various rout path data transfer mechanisms. In [19] authors implement two algorithms - power and delay aware multipath routing protocol (PDMRP) and slow start exponential and liner algorithm (STELA) using cross layer design. STELA adjust sleeping window when there is no activity in the network and thus improves energy efficiency of the network. While PDMRP selects shortest and energy efficient path when there is any network activity. Using these two algorithms, author designed an energy efficient multipath routing protocol using adjustable sleeping window (EMRAS) which reduced overall energy consumption without degrading QoS of service. The limitation of this method is to adjust sleeping window of node. For that node has to continuously 
monitor activity in the network so that it can switch between sleep and awake mode.

In [20] a minimum route power/ maximum battery life (MPR/MBL) scheme has been described. In this scheme authors have calculated route cost by combining a value for transmission and processing power and a separate value for remaining battery energy. By using these separate values minimum power routes are selected when battery energy is high and nodes with less energy are eliminated from the route. The performance of this method degrades in high mobility network. In [21], authors described problem of non-uniform load distribution in MANET. In this a lightweight dynamic channel allocation algorithm using non GPS based synchronization algorithm has been proposed. Also a cooperative load balancing algorithm in which nodes select their channel access providers based on available resources is described. In [22], an efficient power aware routing (EPAR) has been described that improves network lifetime and works in high mobility network. Unlike traditional power control algorithms, EPAR not only uses residual battery power of a node but also uses expected energy spent in reliably forwarding data packets over a specific link to identify capacity of node. Using the min-max formulation EPAR selects the path that has maximum packet capacity at minimum packet transmission capacity. The EPAR calculates expected energy spent in forwarding data packets which causes increase in overhead of the network.

In [23] a threshold based routing protocol is proposed in which the mobile the node selectively choose whether to use dynamic or static protocol based on its velocity. If the velocity of node is very high then it joins the reactive cluster in which all nodes moves very fast. If the velocity of node is slow then it joins proactive cluster where all nodes in cluster move slowly. In this method node has to switch between proactive and reactive cluster and hence cluster has to maintain up to date entries in routing tables. In [24] author proposed reliable and energy efficient multicast routing (REHMRP) for MANET. In this paper, the author designed the Node Reliability Decisive factor based on power level of the node, link stability, and node mobility. The nodes having maximum reliability decisive factor is used in route calculation and stability of link is also based on this factor. REHMRP provides a reliable routing since the nodes present in the network are cheeked against its energy level and link status before deciding the routes to multicast group member. In [25] a Battery Power Management Routing (BPMR) protocol is proposed that archives the viability and maximizing the number of alive devices. BPMR has both of the characteristics of avoiding low battery devices by Minimum Battery Cost Routing (MBCR) and avoiding long participation duration devices. BPMR avoids low battery energy nodes in route selection thus the devices can prolong their battery life and number of available nodes is high in the network. In this method nodes participation time is restriceted.

In [26] a Mobile agent based Energy-efficient reliable routing protocol is proposed which uses link cost and minimum drain rate (MDR) for energy consumption and link availability. In this protocol, the mobile agents are randomly organized and transfer hop-by-hop until the destination is reached. Hence from each hop they traverse, they collect information related to above metrics and a combined list cost metric is estimated based on these metrics. In this method network load is calculated by examining node burden which is tedious task because node can consume energy by not only performing active task but also some inactive internal task hence calculating node burden is increased in overhead of the network. In [27] a Fault tolerant multipath routing protocol is proposed. To reduce the packet loss due to route breakage, a new route discovery mechanism has been used. In this protocol, nodes determine multiple disjoint routes with more battery power and residual energy, to every active destination.

\section{Proposed optimized EE-HRP protocol}

We divide our protocol into different phases asnetwork initialization phase, create zone phase, route request phase and route reply phase. In this we use our previously designed algorithms namely Zone Head Selection Algorithm (ZHSA) which select maximum residual energy node as zone head using max-heap tree algorithm and Node Energy Monitoring Algorithm (NEMA) which monitors individual nodes energy level and assign various roles to nodes based on remaining battery power of node. These two algorithms are mentioned in [28]. The working of EE-HRP explained below:

\subsection{Network initialization phase}

Initial Assumptions:

- All nodes are homogeneous with same capabilities.

- Nodes are equipped with GPS and are location aware. 

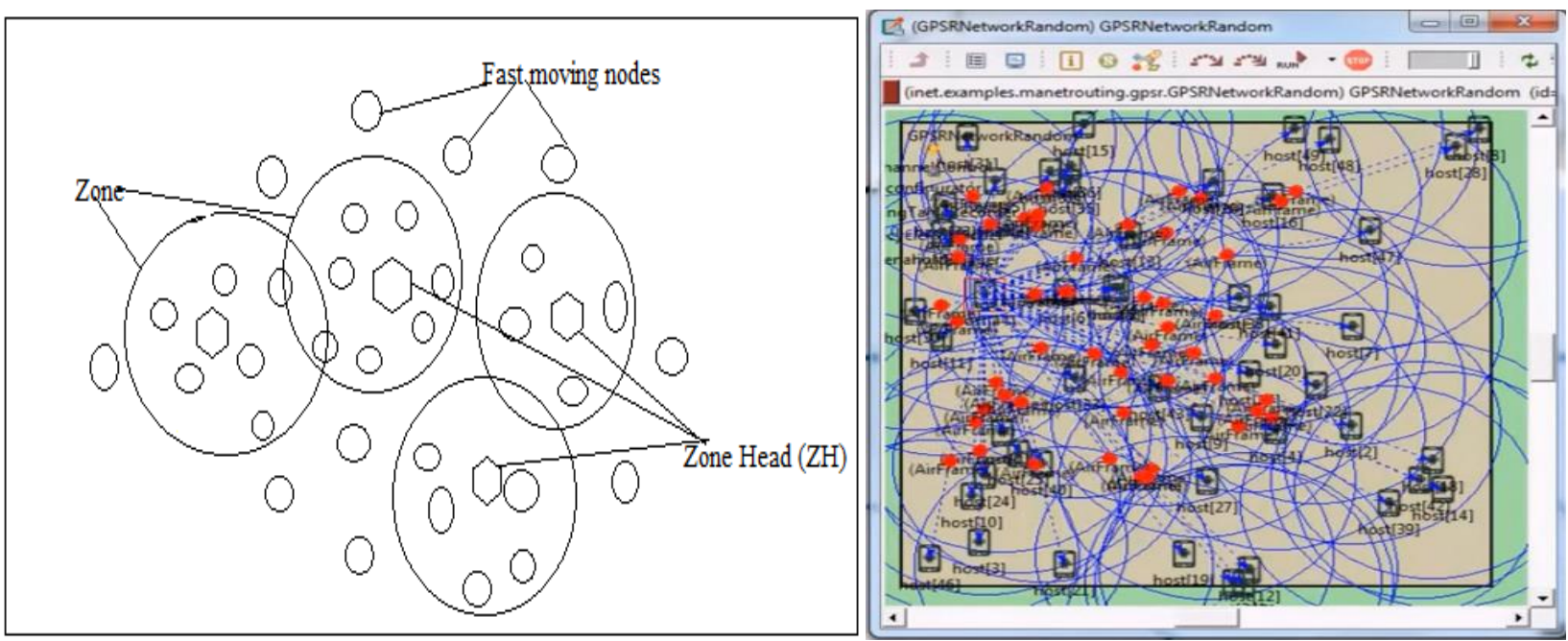

Figure. 2 Zone creation

- Every node is capable of changing its transmission power level depending on the distance to the receiver.

- The network has continuous data to send.

- Links are symmetric. Based on RSSI (Received Signal Strength Indication), any node can compute the approximate distance to another node for a given transmission power.

- Let $\mathrm{G}_{\mathrm{m}}$ is the set of nodes consisting of sources and all forwarding nodes located along $\mathrm{m}^{\text {th }}$ route to destination.

- $\quad \mathrm{ER}_{\mathrm{n}}$ be the residual energy of each node.

- $\mathrm{N}_{\text {speed }}$ is the speed of mobile node.

- $\mathrm{L}_{\mathrm{D}}$ is total data length to be transmitted

- $\mathrm{t}_{\mathrm{c}}$ is the duration of entire data transmission

\subsection{Create zone phase}

The nodes broadcast Zone_REQ message containing node ID, location ID, available residual energy and speed of node. The node finds its neighbor and exchanges location information. The group of nodes having same location ID forms one zone. In Fig. 2, four zones are shown with respective zone heads. The fast moving nodes are not part of any zone. Also, the relative speed of node within zone is same. The zone radius is maintained using two hop distance the simulation result for the same is shown in Fig. 2 above.

If a node does not find any neighbor within two hop distance then that node form new zone and itself act as $\mathrm{ZH}$. The node which has fast moving speed above the threshold, that node is not included in any zone.

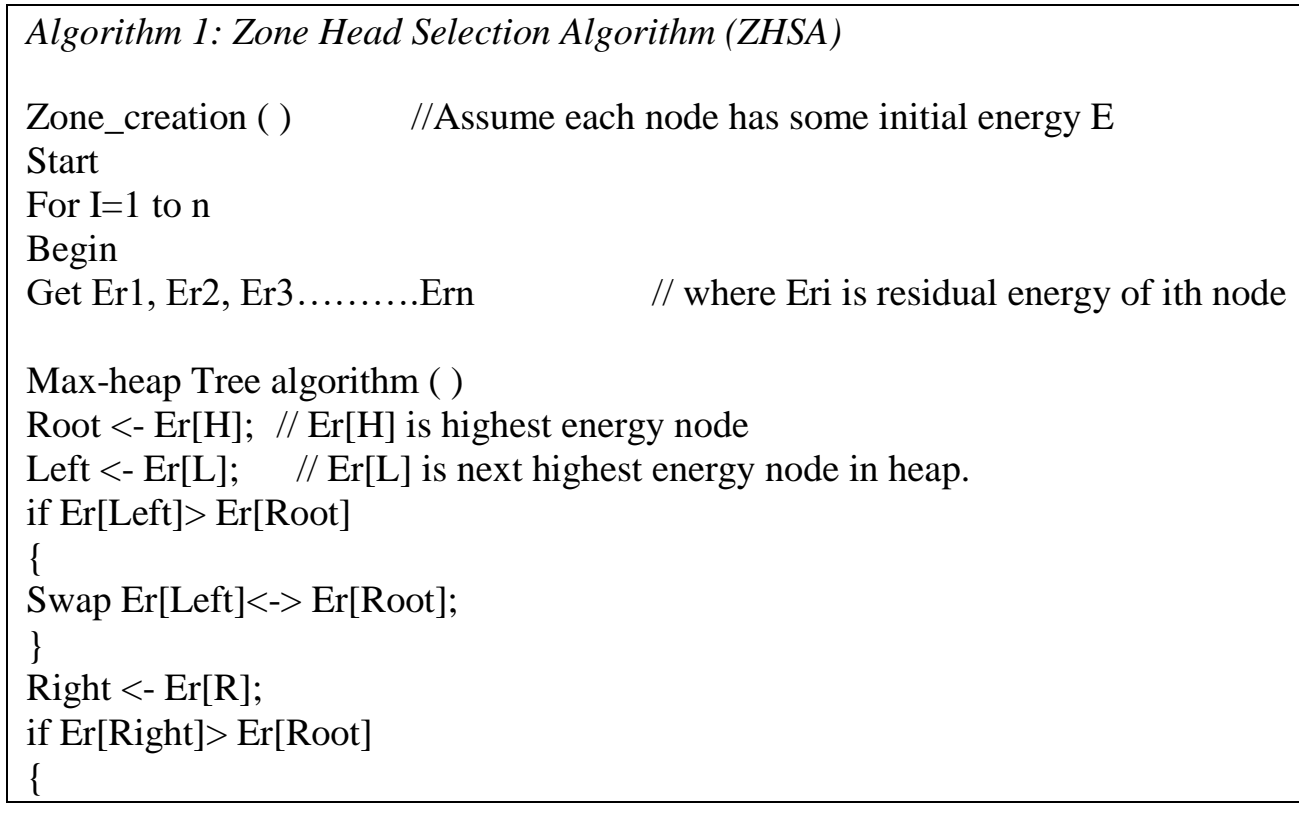


Swap Er[Right] $<->\operatorname{Er}[$ Root $]$;

\}

Repeat until all the nodes within zone comes under max-heap tree

$\operatorname{Er}[\max ]<-\mathrm{ZH}$

// root node of heap is always act as zone head $(\mathrm{ZH})$.

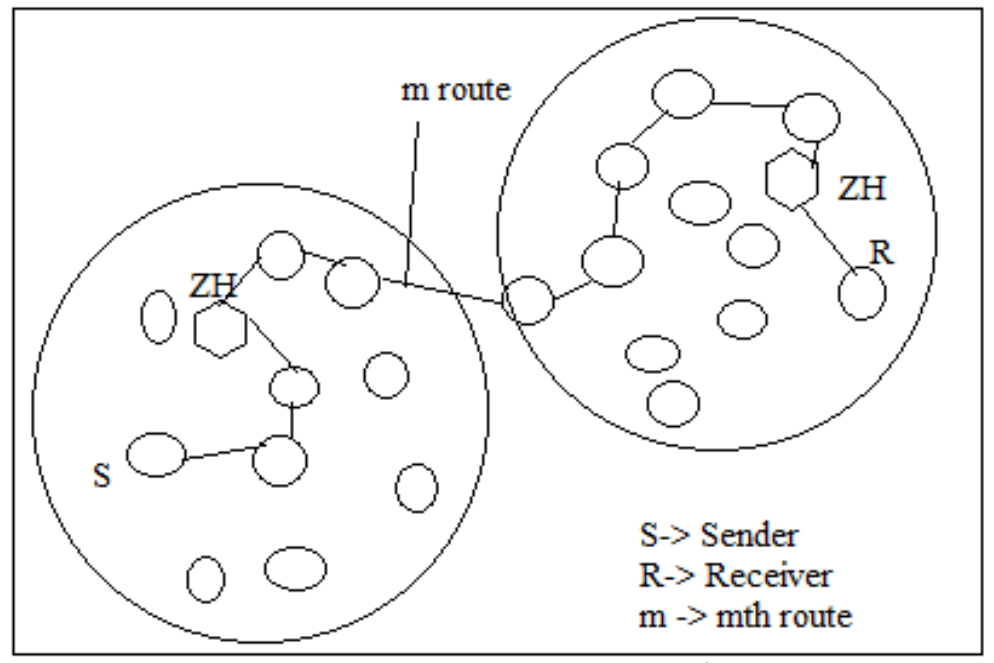

Figure. 3 Total energy across $\mathrm{m}^{\text {th }}$ root

\subsection{Node energy model}

By using ZHSA() function the maximum residual energy node will be selected as zone head.

$$
Z H=E R_{\max }{ }^{n} \text { where } \mathrm{n} \text { belongs to } \mathrm{Gm}
$$

The other nodes are assigned with different roles depending on available residual energy of each node. This is performed by using NEMA() function. Let, energy required to send one bit of packet is given as $\mathrm{e}_{\mathrm{m}}{ }^{\mathrm{n}}$. Total transmission energy required to send one bit along route $m$ (as shown in Fig. 3) is given by

$$
E m=\sum e_{m}{ }^{n}
$$

Total transmission energy $e_{n}$ that is required by node $\mathrm{n}$ to transmit data bits is given as

$$
e_{n}=\sum q_{n}^{r} e_{n}^{r}
$$

Where,

- $\mathrm{q}_{\mathrm{n}}{ }^{\mathrm{r}}$ is remaining number of data bits for ongoing connection routed through node $n$ along $\mathrm{r}^{\text {th }}$ route.

- $e_{n}{ }^{r}$ is transmission energy that node $n$ is required to transmit one data bit along route.
We assumed that all nodes start with a finite amount of battery power and energy dissipation per bit of data and control packet transmission and reception are known. As shown in fig.3, we calculate minimum effective energy remaining in all nodes along $\mathrm{m}^{\text {th }}$ transmission route as

$$
E R_{\min }=\min \left(E R^{n}-e_{n}-L_{D} e_{m}{ }^{n}\right)
$$

Where,

- $\quad \mathrm{ER}_{\min }$ is the minimum residual energy of node $\mathrm{n}$.

- $\mathrm{e}_{\mathrm{n}}$ is total transmission energy that is required by node $\mathrm{n}$ to transmit number of data bits along the route.

- $\mathrm{L}_{\mathrm{D}}$ is total data size to be transmitted.

- $\quad \mathrm{e}_{\mathrm{m}}{ }^{\mathrm{n}}$ is transmission energy required to send a bit of data from node $\mathrm{n}$ to next downstream node along $\mathrm{m}^{\text {th }}$ route.

The $\mathrm{m}^{\text {th }}$ route need not be the shortest path between sender and receiver, but it is the route which gives maximum effective energy route i.e. all the nodes in this $\mathrm{m}^{\text {th }}$ route has maximum residual energy.

We create NEMA which calculate this $\mathrm{ER}_{\text {min }}$ as shown in eq.4 and check it against different threshold levels. NEMA () function assigned various roles to the nodes depending upon available residual energy as given in Table 1 below: 
Table 1. Various roles assigned to nodes by NEMA ( )

\begin{tabular}{|c|c|}
\hline Remaining battery power & Duties performed by node \\
\hline $80 \%-100 \%$ & Zone head $(\mathrm{ZH})$ \\
\hline $60 \%-80 \%$ & Relay $(\mathrm{RN})$ \\
\hline $40 \%-60 \%$ & Transmit and Receive data $(\mathrm{CN})$ \\
\hline $30 \%-40 \%$ & Urgent data transmission as a relay node $(\mathrm{EM})$ \\
\hline $0 \%-30 \%$ & Periodic sleep mode $(\mathrm{SN})$ \\
\hline
\end{tabular}

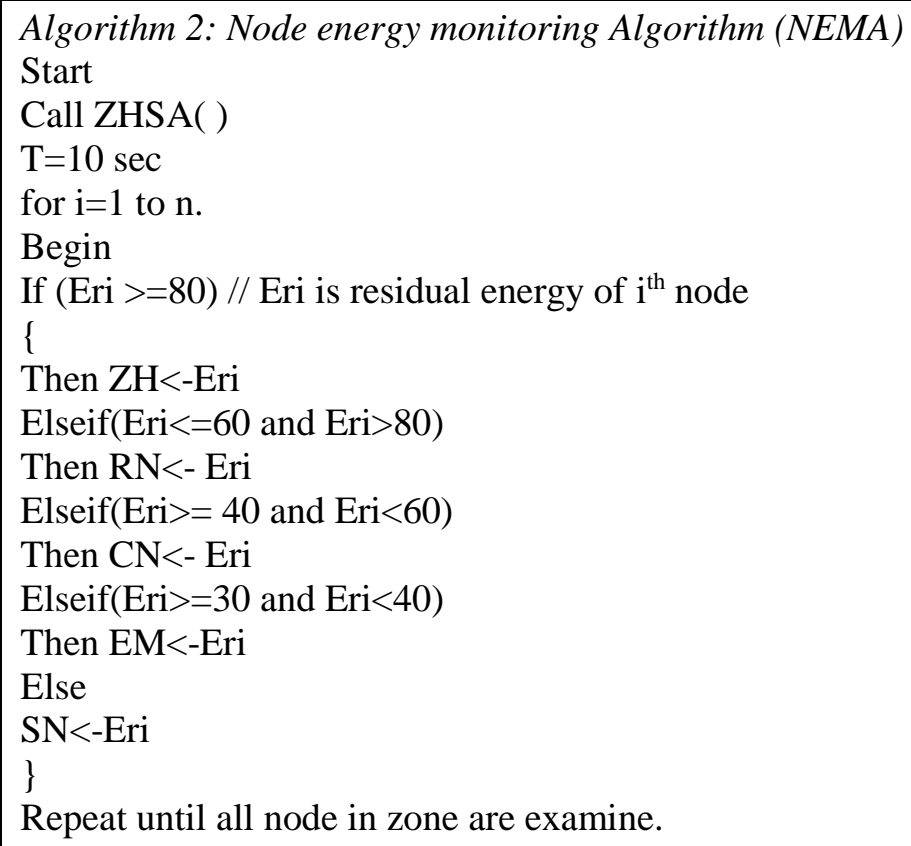

Algorithm 3: NodeSpeedModel()

step1: Assume the random speed of all nodes in the networks

step2: Consider speed threshold $\mathrm{TH}_{\mathrm{s}}$ at $30 \mathrm{~m} / \mathrm{s}$.

Step3: If $\mathrm{N}_{\text {speed }}$ is below $\mathrm{TH}_{\mathrm{s}}$ then node relays packet.

Elseif speed is between $30-50 \mathrm{~m} / \mathrm{s}$ then node relys packet with medium priority $\mathrm{P} 2$.

Elseif speed is between $50-60 \mathrm{~m} / \mathrm{s}$ then node relys packet with high priority $\mathrm{P} 1$

Else node never participate in packet relay.

\subsection{Node speed model}

In optimized EE-HRP we consider the random speed of all nodes in the network. We create Node Speed Monitoring Algorithm (NSMA) to monitor the speed of node. Here we use speed threshold $\mathrm{TH}_{\mathrm{s}}$ at $30 \mathrm{~m} / \mathrm{s}$.

- If $\mathrm{N}_{\text {speed }}$ is below $\mathrm{TH}_{\mathrm{s}}$ then node relay packet.
- If it is above threshold then we use priority levels to forward the data packets as shown in Algorithm 3.

\subsection{Route discovery phase}

The route discovery phase is divided into two modules, viz.

i. Intra-zone routing

ii. Inter-zone routing 


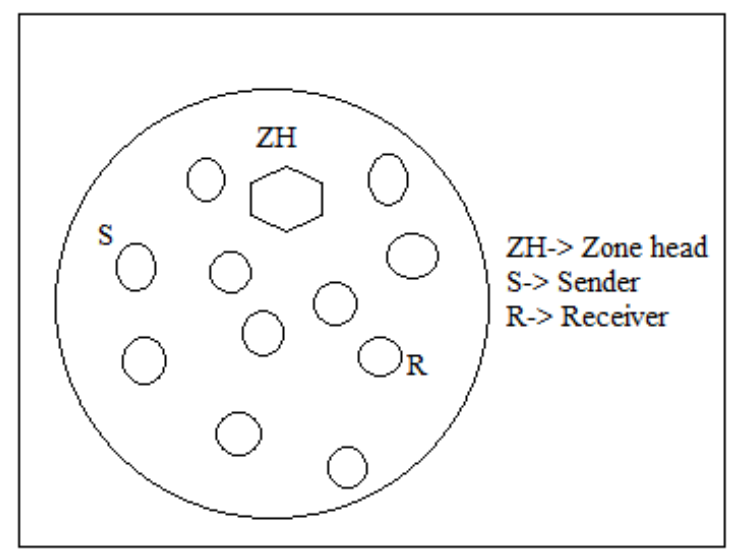

Figure. 4 Intra-zone routing

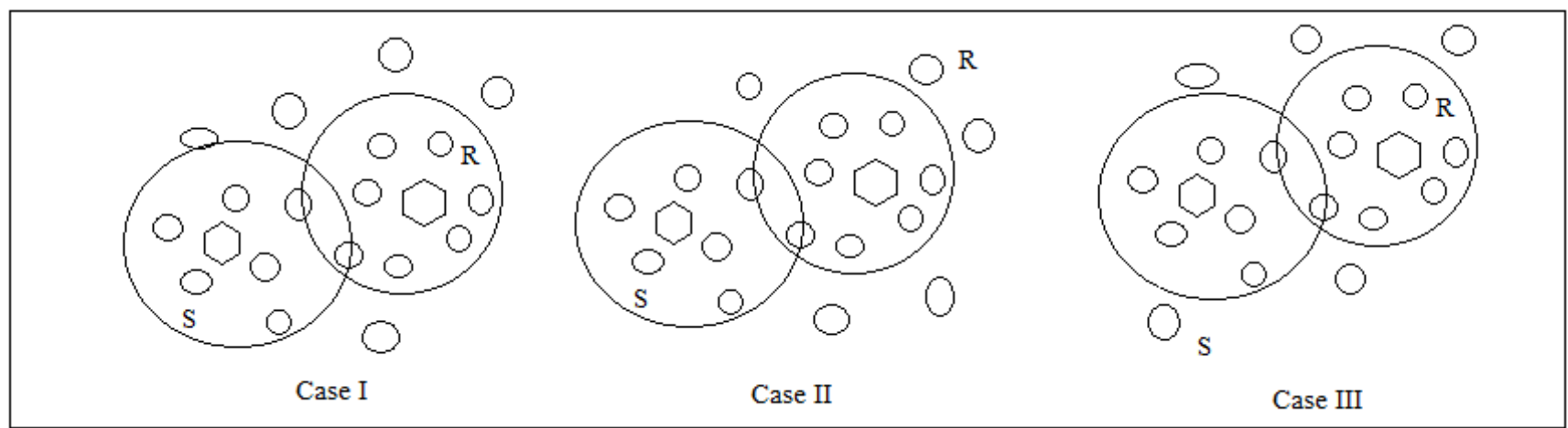

Figure. 5 Inter-zone routing

\section{i. Intra-zone routing}

At the time of route discovery, RREQ packet is broadcasted by source S. The header of RREQ packet includes <source_id, destination_id, hop_count, $\mathrm{LT}_{\mathrm{m}}, \mathrm{Er}_{\mathrm{min}}, \mathrm{N}_{\text {sleep }}, \mathrm{N}_{\text {speed }}>$. Where, $\mathrm{LT}_{\mathrm{m}}$ is a lifetime of $\mathrm{m}^{\text {th }}$ route, $\mathrm{N}_{\text {sleep }}$ is a count of nodes in sleep mode and $\mathrm{N}_{\text {speed }}$ is speed of node.. Initially $\mathrm{LT}_{\mathrm{m}}=0$ and $\mathrm{N}_{\text {sleep }}=0$. If a node wants to send $\mathrm{a}$ packet, then, first of all, it checks whether the destination node is present in its neighbor table or not. If the destination node is on the table that means both nodes are within the same zone and having same relative speed and hence intra-zone routing is performed (As shown in Fig. 4). The source node will route the packet directly to destination node using any reactive routing like AODV, DSR etc.

\section{ii. Inter-zone routing}

If destination node is not present in the neighbor table it means both the nodes are in different zone and hence inter-zone routing is performed by using zone head. The inter-zone requires following cases to be considered.

CASE I: If source and destination nodes are in the different zone
As shown in Fig. 5 (case I), the source node sends RREQ packet via respective zone head $\mathrm{ZH}$. The ZH accept RREQ, check destination ID and $\mathrm{N}_{\text {speed }}$ and forward packet to all available $\mathrm{ZH}$. The other ZH accepts RREQ, check destination ID and if destination is in the same zone then forward packet to destination node otherwise discard the packet.

\section{CASE II: if source is in zone and destination is not in any zone}

In this case, source send RREQ packet to $\mathrm{ZH}$ which checks destination ID and forward packet to all zones. As shown in Fig. 5 (case II) the destination is not found in any zone because node having high speed is not included in any zone and hence packet is discarded by all ZHs.

\section{CASE III: if source is not in any zone and destination is in zone}

In this case source broadcast RREQ packet. The nodes who receive this RREQ packet check location ID and speed of node. If speed is above threshold and is in the range $30-50 \mathrm{~m} / \mathrm{s}$ then node forward packet with priority $\mathrm{P} 2$. If speed is in the range 50$60 \mathrm{~m} / \mathrm{s}$ then packet is forwarded with priority P1. 


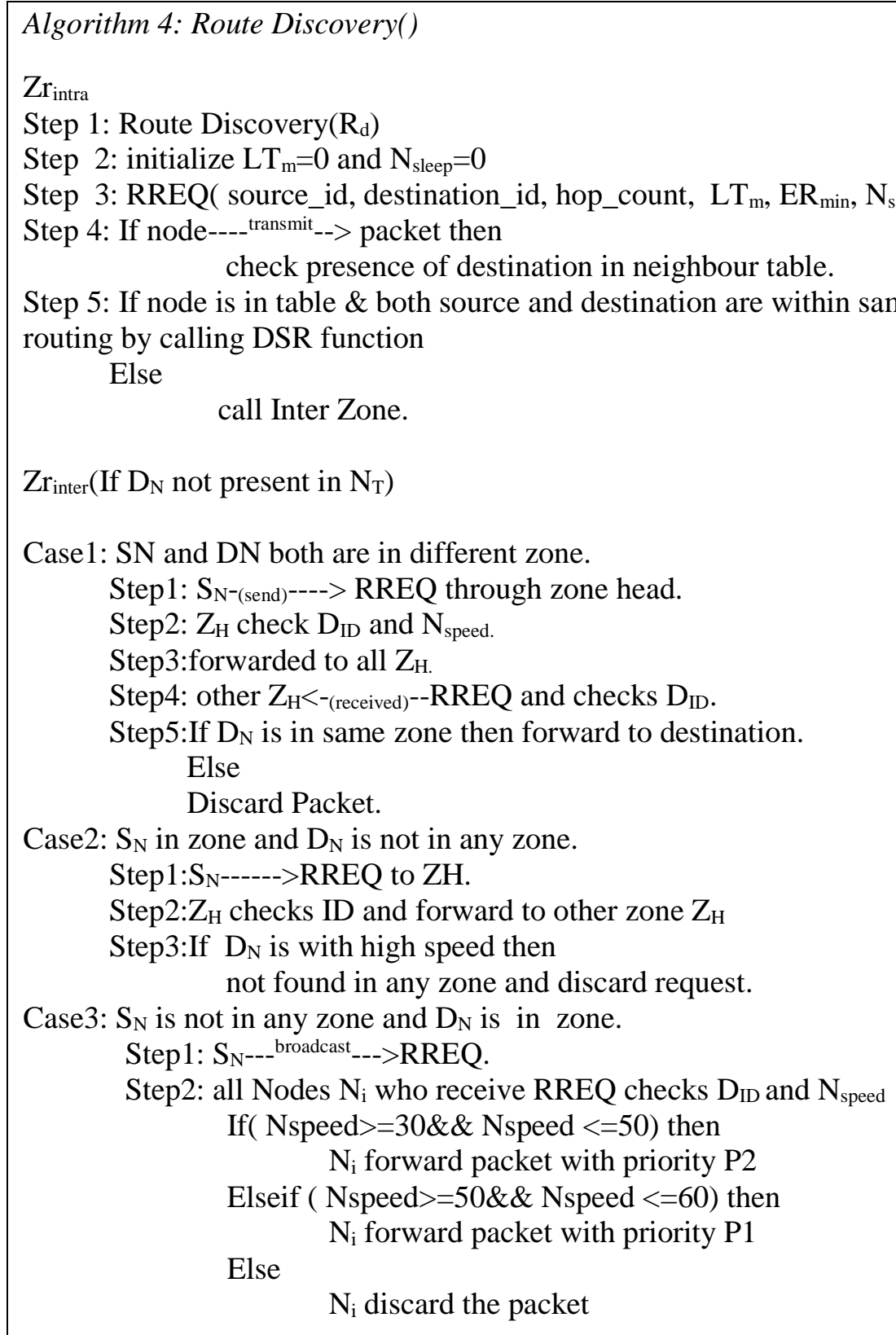

And if speed is above $60 \mathrm{~ms}$ node discard the packet. This is shown in Fig. 5 (Case III).

As RREQ packet travels along route $m$, its value is updated as follows

$$
\begin{aligned}
& \text { if }\left(E R_{\text {min }}=E R_{S N}\right) \text { then } \\
& N_{\text {sleep }}=N_{\text {sleep }}+1 \\
& \text { else if }\left(E R_{\text {min }}=E R_{S N}<E R_{E M}<E R_{C N}\right) \& \&
\end{aligned}
$$

$($ Priority $=P 1)$ then

$$
L T_{m}=L T_{m}+1
$$$$
\text { else if }\left(E R_{\min }=E R_{R N}\right) \& \&
$$

$($ Priority $=P 2)$ then

$$
L T_{m}=L T_{m}+3
$$

The destination node waits for certain time $\mathrm{T}_{\text {wait }}$ till all RREQ meant for the destination is reached. The destination nodes compares dest_seq_no, hop_count and $\mathrm{LT}_{\mathrm{m}}$ of a route in RREQ packet received with dest_seq_no, hop_count and $\mathrm{LT}_{\mathrm{m}}$ of a route in routing table and then selects the route with maximum energy and minimum number of sleep nodes as

$$
\begin{aligned}
& \text { for all RREQ, } \\
& \text { reject RREQ's with } \mathrm{N}_{\text {sleep }}>=3
\end{aligned}
$$

The destination node then calculate following parameter for all remaining RREQ packet

$$
R_{f}^{m}=L T_{m} / \text { hop_count }
$$




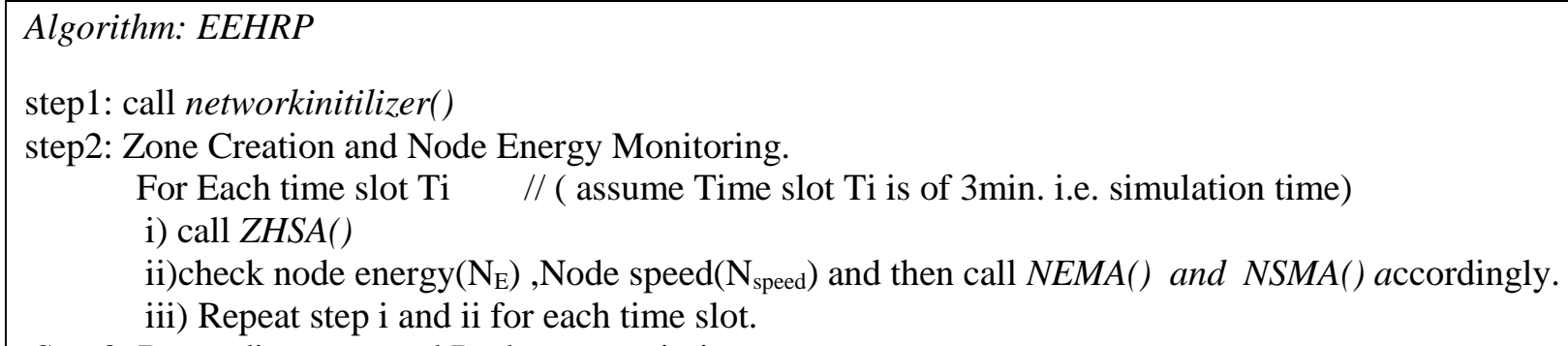

Step3: Route discovery and Packet transmission

If routing is within zone then

i) call Zintra()

ii) call NodeSpeed()

Else if routing is in different node.

i) call Zinter()

ii) call NodeSpeed()

Step4: Repeat step 2 and step 3 until the simulation end.

Where $R_{f}^{m}$ value indicates whether $m^{\text {th }}$ route is selected or not.

The destination node then selects route with maximum $\mathrm{R}_{\mathrm{f}}{ }^{\mathrm{m}}$ and changes are made in routing table.

\subsection{Route reply phase}

Once the route is discovered, the nodes in the route will send route_reply messages in which nodes acknowledge the selected route by sending their location information speed along the reverse path.

- The selected nodes in the path send out Route_reply.

- Each selected node add its location (x,y) and speed Nspeed in route_reply.

- These location parameters are used by each sending node to find the distance between next hop node and itself.

- Nspeed value is used to set priority of sending reply based on the motion of the node.

The minimum transmission power required is then adjusted by sender node by using the distance between the current node and next hop node.

We summarize the complete optimized EE-HRP algorithm as above:

\section{Performance evaluation}

We have carried out the performance evaluation of our work using Omnet++ simulator. We have compared working of EE-HRP against protocols standard protocols like Power aware Localized Routing (PLR) [11] which comes under transmission power control approach, Localized Energy Aware Routing (LEAR) Protocol [13], which comes under load distribution approach and SPAN protocol [15] which comes under sleep/power down approach of energy efficient routing for MANET.

We have used various performance metrics like packet delivery ratio, packet drop, routing overhead, total power consumed and average remaining battery power. For simulation we consider two scenarios. In first network scenario we consider maximum movement speed of nodes and check effect of node mobility on optimized EE-HRP. And in second scenario we vary number of nodes and check the performance of EE-HRP in small and large network.

The different parameter setting is shown in Table 2 below.

Table 3 shows value for number of packet sent, lost, number of routing packets and energy consumed in network when speed of node is varying. Fig. 6 shows effect of node speed on packet delivery ratio, packet drop, routing overhead and energy consumption.

\subsection{Packet delivery ratio}

It is the ratio of a number of data packets received by the number of data packets sent as shown in Eq. (6).

$$
P D R=P_{R} / P_{T}
$$

Where, PDR -> Packet Delivery ratio, $\mathrm{P}_{\mathrm{R}}->$ number of packet received and $\mathrm{P}_{\mathrm{T}} \rightarrow$ number of packet transmitted.

Fig. 6 (a) and 7 (a) show comparison of packet delivery ratio. In Fig. 6 (a), we see that EE-HRP 
Table 2. Simulation parameters

\begin{tabular}{|l|l|}
\hline Simulation parameters & Simulation values \\
\hline Simulation Time & $0-100 \mathrm{sec}$ \\
\hline MANET standard & IEEE $802.11 \mathrm{e}$ \\
\hline Number of nodes & 100 \\
\hline Base protocol & ZRP \\
\hline System Bandwidth & $2 \mathrm{Mbps}$ \\
\hline Traffic type & $\mathrm{CBR}$ \\
\hline Packet Size & 512 bytes \\
\hline Node Mobility & $0-100 \mathrm{~m} / \mathrm{s}$ \\
\hline Transmission power & $2.0 \mathrm{~mW}$ \\
\hline Reception power utilized & $5.0 \mathrm{~mW}$ \\
\hline Simulation Environment & $1500 \times 1500$ \\
\hline Channel Propagation & Wireless / Two ray Ground \\
\hline
\end{tabular}

Table 3. Values for number of packet sent, lost, number of routing packets and energy consumed in network for different speed of node.

\begin{tabular}{|c|c|c|c|c|c|}
\hline $\begin{array}{l}\text { Max. } \\
\text { movement } \\
\operatorname{speed}(\mathrm{m} / \mathrm{s})\end{array}$ & $\begin{array}{l}\text { Routing } \\
\text { Protocol }\end{array}$ & Sent packet & $\begin{array}{l}\text { Routing } \\
\text { Packet }\end{array}$ & Lost Packet & $\begin{array}{l}\text { Remaining } \\
\text { Energy }\end{array}$ \\
\hline \multirow{4}{*}{10} & EE-HRP & 3528 & 270 & 63 & 7056 \\
\hline & PLR & 3743 & 330 & 48 & 7486 \\
\hline & LEAR & 3511 & 390 & 74 & 7021 \\
\hline & SPAN & 3745 & 293 & 52 & 7490 \\
\hline \multirow{4}{*}{20} & EE-HRP & 3629 & 320 & 69 & 7258 \\
\hline & PLR & 3798 & 400 & 52 & 7596 \\
\hline & LEAR & 3625 & 498 & 83 & 7251 \\
\hline & SPAN & 3726 & 336 & 92 & 7552 \\
\hline \multirow{4}{*}{40} & EE-HRP & 3581 & 1038 & 85 & 7362 \\
\hline & PLR & 3723 & 857 & 82 & 7646 \\
\hline & LEAR & 3696 & 1179 & 119 & 7378 \\
\hline & SPAN & 3875 & 648 & 136 & 7650 \\
\hline \multirow{4}{*}{80} & EE-HRP & 3682 & 1038 & 127 & 7564 \\
\hline & PLR & 3793 & 857 & 138 & 7786 \\
\hline & LEAR & 3589 & 1179 & 152 & 7591 \\
\hline & SPAN & 3895 & 462 & 168 & 7790 \\
\hline
\end{tabular}

performs comparatively better because network will not forward RREQ packet unless it finds minimum path having maximum residual energy nodes in it. Also speed of all nodes, whether it may be the sender, receiver or relay node is considered while selecting routes. High speed nodes and low energy nodes are not participated in data transmission process. Hence packet drop is less which increases the delivery ratio of the network. In Fig. 7 (a) we see that Optimized EE-HRP efficiently delivered packets $(85 \%-90 \%)$ when load is less but as traffic increases the performance degrades slowly because packets are dropped due to congestion.

\subsection{Packet drop}

This means a number of packets deleted in the network. Fig. 6 (b) and 7 (b) show that packet drop is less in EE-HRP as it reduces flooding of RREQ and also there is less packet drop due to link breakages and collision. Fig. 6 (b) shows that the packet drop is increased if the mobility of nodes increased in the given network. Fig. 7 (b) indicates that as traffic is increases, congestion is occurred due to which node drops packet hence the packet drop is increased when traffic or load is increased in network. 


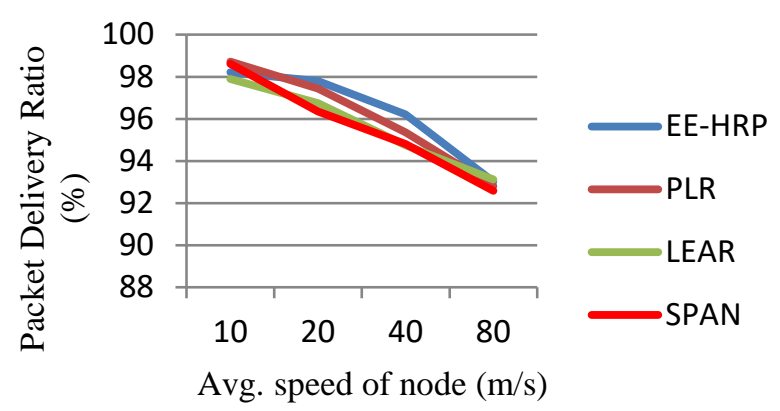

(a)

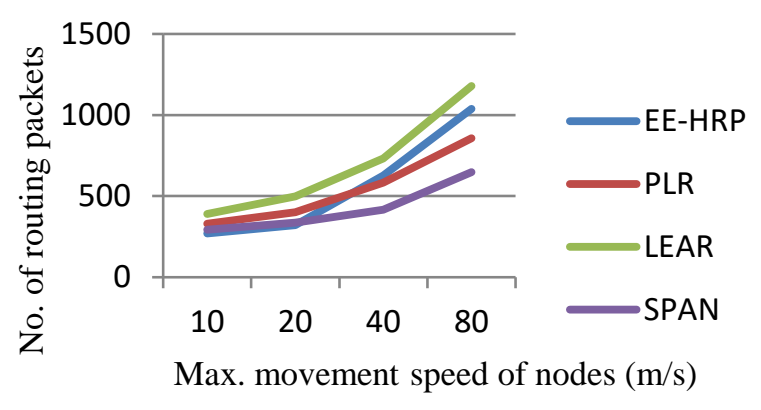

(c)

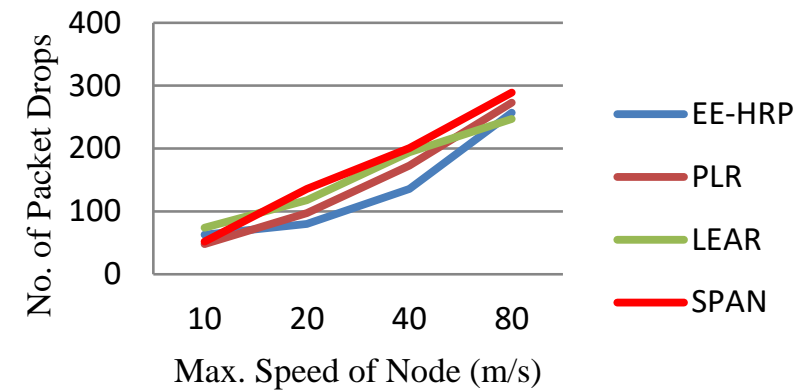

(b)

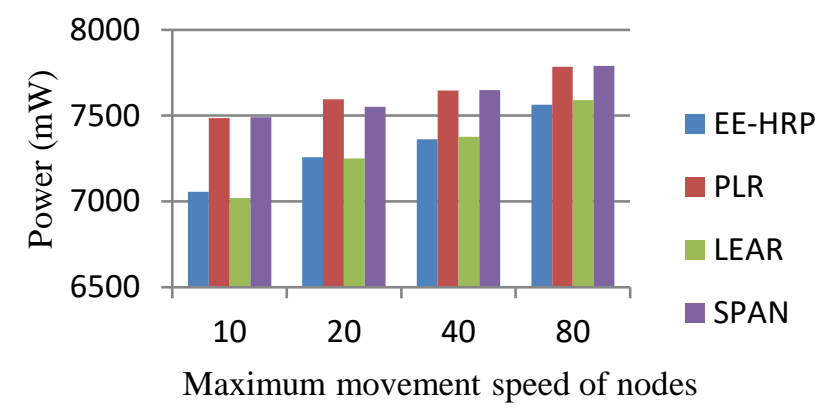

(d)

Figure. 6 Effect of speed of nodes on: (a) Packet Delivery Ratio, (b) Packet dropped, (c) Routing overhead, and (d) Power consumption

\subsection{Routing overhead}

The routing packets do not carry any application content like data packets do. Both, routing and data packets have to share the same network bandwidth most of the times, and hence, routing packets are considered to be an overhead in the network. This overhead is called routing overhead. Fig. 6 (c) and Fig. 7 (c) shows a comparison of routing overhead of EE-HRP with different protocols. It shows that routing overhead is less in EE-HRP due to less flooding and reboardercast. Fig. 6 (c) shows that as the speed of node increases the overhead is also increased to some extent. Fig. 7 (c) shows that as the number of nodes increases the overhead is also increased because there is more traffic in network and to route the traffic more messages has to pass through the network.

\subsection{Total Power Consume}

It is the amount of battery power consumed by a node after transmission, reception or relaying messages.Let Et is energy required for transmitting packet, Er is energy required for receiving a packet then total energy consumed $E_{C}$ is given as

$$
E_{C}=E t+E r
$$

If node sends $\mathrm{N}$ number of packets at given time and each packet is of length $\mathrm{L}$, then energy required to send one packet (Ep) is given as

$$
E p=N L_{p} e_{m}^{n}
$$

Where,

- $\quad \mathrm{L}_{\mathrm{p}}$ is total data size to be transmitted.

$\circ \quad \mathrm{e}_{\mathrm{m}}{ }^{\mathrm{n}}$ is transmission energy required to send a bit of data from node $\mathrm{n}$ to next downstream node along $\mathrm{m}^{\text {th }}$ route.

Hence we modify Eq. (7), which gives total energy consumed by a node to transmit, receive or rely $\mathrm{N}$ number of packets as

$$
E_{C}=E t E p+E r E p
$$

The Fig. 6 (d) and Fig. 7 (d) indicate that total power consumed by EE-HRP is comparatively less when speed of node is slow but as speed increase more power is consumed as a node has to compute new routes and has to retransmit packets because of congestion. 


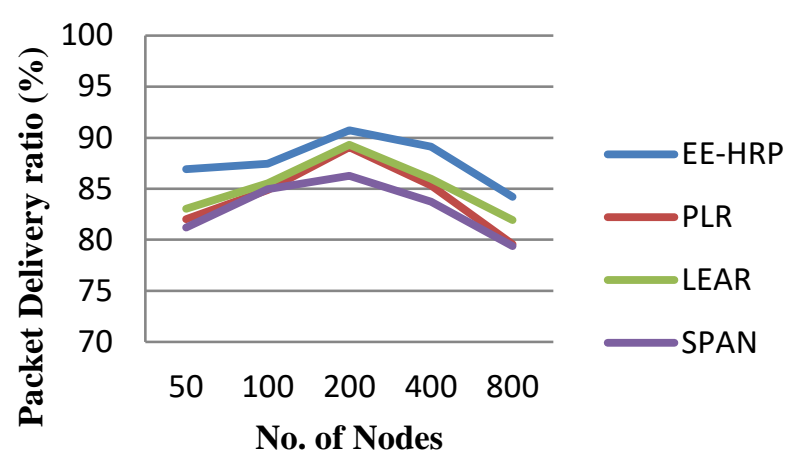

(a)

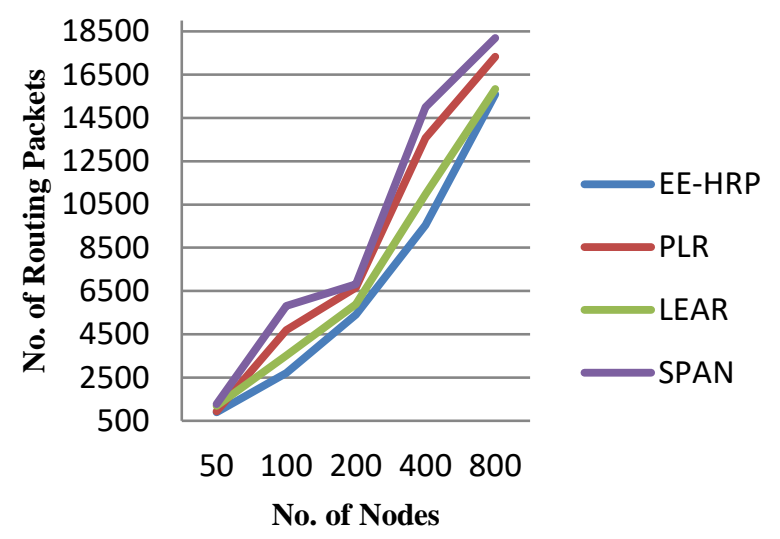

(c)

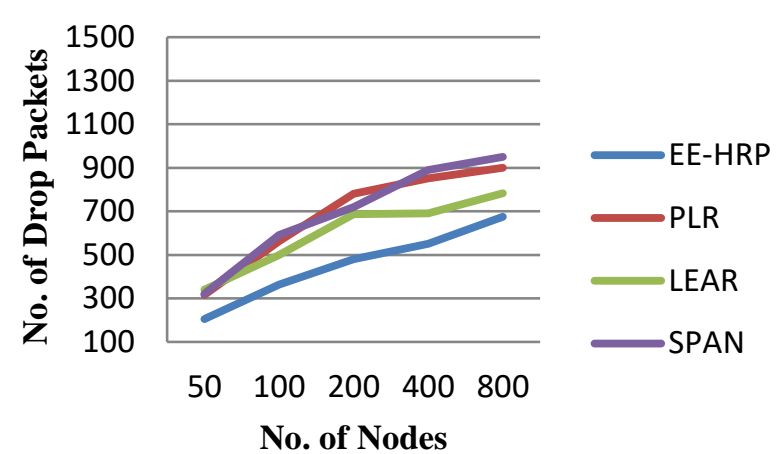

(b)

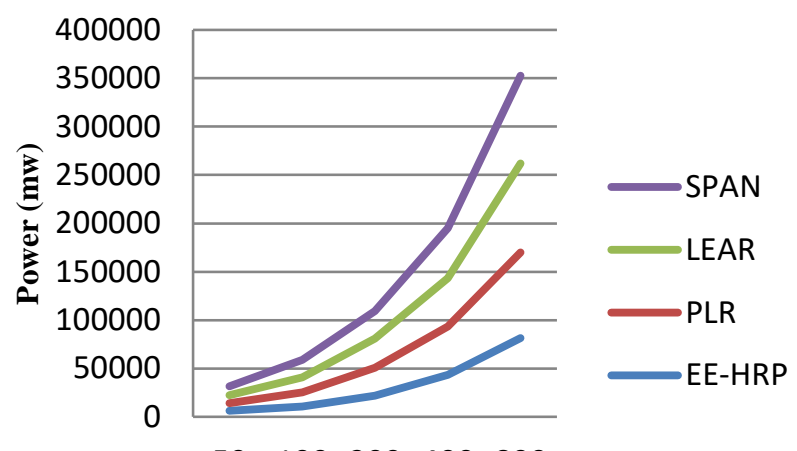

$\begin{array}{lllll}50 & 100 & 200 & 400 \quad 800\end{array}$

No. of Nodes

(d)

Figure. 7 Effect of number of nodes in network on: (a) Packet delivery Ratio, (b) Packet dropped, (c) Routing overhead, and (d) Power consumption

Table 4. Values for number of packet sent, lost, number of routing packets and energy consumed in network for different number of node

\begin{tabular}{|c|c|c|c|c|c|c|}
\hline $\begin{array}{c}\text { No. of } \\
\text { nodes }\end{array}$ & $\begin{array}{c}\text { Routing } \\
\text { Protocol }\end{array}$ & $\begin{array}{c}\text { Sent } \\
\text { packet }\end{array}$ & $\begin{array}{c}\text { Routing } \\
\text { Packet }\end{array}$ & $\begin{array}{c}\text { Lost } \\
\text { Packet }\end{array}$ & $\begin{array}{c}\text { Receive } \\
\text { packet }\end{array}$ & $\begin{array}{c}\text { Energy } \\
\text { Consumed }\end{array}$ \\
\hline \multirow{4}{*}{50} & EE-HRP & 1568 & 893 & 205 & 1363 & 6244 \\
\cline { 2 - 7 } & PLR & 1582 & 935 & 316 & 1266 & 7862 \\
\cline { 2 - 7 } & LEAR & 1549 & 1190 & 340 & 1209 & 8254 \\
\cline { 2 - 7 } & SPAN & 1543 & 1274 & 321 & 1222 & 9158 \\
\hline \multirow{4}{*}{100} & EE-HRP & 3692 & 2713 & 363 & 3329 & 10726 \\
\cline { 2 - 7 } & PLR & 3719 & 4696 & 562 & 3157 & 14639 \\
\cline { 2 - 7 } & LEAR & 3725 & 3519 & 500 & 3225 & 15591 \\
\cline { 2 - 7 } & SPAN & 3846 & 5816 & 592 & 3254 & 18100 \\
\hline \multirow{4}{*}{200} & EE-HRP & 6253 & 5398 & 480 & 5773 & 21770 \\
\cline { 2 - 7 } & PLR & 6384 & 6662 & 780 & 5604 & 29053 \\
\cline { 2 - 7 } & LEAR & 6298 & 5882 & 686 & 5612 & 29843 \\
\cline { 2 - 7 } & SPAN & 6371 & 6815 & 720 & 5651 & 28787 \\
\hline \multirow{4}{*}{400} & EE-HRP & 12167 & 9553 & 550 & 11617 & 43547 \\
\cline { 2 - 7 } & PLR & 12735 & 13576 & 852 & 11883 & 49641 \\
\cline { 2 - 7 } & LEAR & 12587 & 10967 & 690 & 11897 & 50366 \\
\cline { 2 - 7 } & SPAN & 12376 & 14989 & 890 & 11486 & 51294 \\
\hline \multirow{4}{*}{800} & EE-HRP & 21687 & 15593 & 675 & 21012 & 81409 \\
\cline { 2 - 7 } & PLR & 21984 & 17327 & 900 & 21084 & 88543 \\
\cline { 2 - 7 } & LEAR & 22987 & 15836 & 783 & 22204 & 91986 \\
\cline { 2 - 7 } & SPAN & 22578 & 18192 & 950 & 21628 & 90756 \\
\hline
\end{tabular}




\subsection{Average remaining power}

It is the amount of battery power remaining in node after transmission, reception or relaying messages.

Table 4 shows value for number of packet sent, lost, number of routing packets and energy consumed in network when number of node varying. Fig. 7 shows effect of number of node on packet delivery ratio, packet drop, routing overhead and energy consumption.

\section{Conclusion}

The Optimized Energy-Efficient Hybrid Routing Protocol (EE-HRP) is designed and implemented. The working of this proposed optimized algorithm EE-HRP is examined against protocol like PER, LEAR and SPAN. The result shows that EE-HRP perform better in low movement speed of nodes (Packet delivery ratio is up to $98 \%$ ), and give average performance in highly mobile network (Packet delivery ratio is up to 93\%), it consumes less energy (average remaining residual battery energy is $20 \%$ more compared to other protocols and network partition is delayed by 40 seconds before the first node drains energy) and have less routing overhead (it is reduced by $7 \%$ ). Proposed algorithm is also performing better in less dense as well as in denser networks. Thus from this analysis, we have concluded that proposed protocol will be able to perform well in static mobility network and maximize network lifetime to considerable extent. In future work we extend this work by increasing speed of nodes so that it works for highly mobile network like VANET.

\section{References}

[1] S. Singh and S. Das, "Survey on Energy Efficient Routing Protocols in Mobile Ad Hoc Networks", World Academy of Science, Engineering and Technology International Journal of Computer, Electrical, Automation, Control and Information Engineering, Vol. 8, No. 2, pp. 347-357, 2014.

[2] Y. Khamayseh, G. Obiedat, and M. Yassin, "Mobility and Load aware Routing protocol for ad hoc networks", Journal of King Saud University-Computer and Information Sciences, Elsevier B.V., Vol 23, pp. 105-113, 2011.

[3] C. Yu, B. Lee, and H. Y. Youn, "Energy Efficient Routing Protocols for Mobile Ad Hoc Network", Wirel. Commun. Mob. Comput. Vol. 3, pp. 959-973, 2003.
[4] S. Ghode and K. Bhoyar, "A Survey on Energy Efficient Routing Algorithms for Ad-Hoc Network", International Journal of Computer Applications, Vol. 67, No. 20, pp. 44-50, 2013.

[5] S. Lim, C. Yu, and C. Das, "RandomCast: An Energy-Efficient Communication Scheme for Mobile Ad Hoc Networks", IEEE Transactions on Mobile Computing, Vol. 8, No. 8, pp. 10391051, 2009.

[6] B. Olagbegi and M. Natarajan, "A Review of The Energy Efficient And Secure Multicast Routing Protocols For Mobile Ad Hoc Networks", International Journal on Applications of Graph Theory in Wireless Ad Hoc Networks and Sensor Networks, Vol. 2, No. 2, pp. 1-15, 2010.

[7] S.-H. Wu, J.-P Sheu, C.-T. King, "Unilateral Wakeup for Mobile Ad Hoc Networks with Group Mobility”, IEEE Transactions on Mobile Computing, Vol. 12, No. 12, pp. 2507-2517, 2013.

[8] N. Vassileva and F. Barcelo-Arroyo, "A Survey of Routing Protocols for Maximizing the Lifetime of Ad Hoc Wireless Networks", International Journal of Software Engineering and Its Applications, Vol. 2, No. 3, pp. 77-90, 2008.

[9] C. Weng, C-W. Chen, P. Chen, and K. C. Chang, "Design of an energy-efficient cross-layer protocol for mobile ad hoc networks", IET Commun., Vol. 7, No. 3, pp. 217-228, 2013.

[10] B. Tavli and W. Heinzelman, "EnergyEfficient Real-Time Multicast Routing in Mobile Ad Hoc Networks", IEEE Transactions On Computers, Vol. 60, No. 5, pp. 707-722, 2011.

[11] I. Stojmenovic and X. Lin, "Power-Aware Localized Routing in Wireless Networks", IEEE Trans. Parallel Dist. Sys., Vol. 12, No. 11, pp. 1122-33, 2001.

[12] J. Zhu and X. Wang, "Model and Protocol for Energy-Efficient Routing over Mobile Ad Hoc Networks", IEEE Transactions on Mobile Computing, Vol. 10, No. 11, pp. 1546-1557, 2011.

[13] K. Woo, C. Yu, H.Y. Youn, and B. Lee, "Nonblocking, localized routing algorithm for balanced energy consumption in mobile ad hoc networks", In: Proc. of Int. Symposium on Modeling, Analysis and Simulation of Computer and Telecommunication Systems, pp.117-124, 2001.

[14] W. C.-W. Tan, S. Bose, and T.-H. Cheng, "Power and mobility aware routing in wireless 
ad hoc Networks", IET Commun., Vol. 6, No. 11 , pp. $1425-1437,2012$

[15] B. Chen, R. Morris, and H. Balakrishnan, "Span: An energy-efficient coordination algorithm for topology maintenance in ad hoc wireless networks", ACM Wireless Networks Journal, Vol. 8, No. 5, pp. 481-494, 2002.

[16] S. Shivashankar, G. Varaprasad, and S. Narayanagowda, "Implementing a new power aware routing algorithm based on existing dynamic source routing protocol for mobile ad hoc networks", IET Network., Vol. 3, No. 2, pp. 137-142, 2014.

[17] W. Tan, S. Bose, and T.-H. Cheng, "Power and mobility aware routing in wireless ad hoc Networks", IET Communication., Vol. 6, No. 11, pp. 1425-1437, 2012.

[18] R. Valikannu, A. George, and S. Srivatsa, "A Novel Energy Consumption Model using Residual Energy Based Mobile Agent Selection Scheme (REMA) in MANETs", In: Proc. of the 2nd International Conference on Signal Processing and Integrated Networks, pp. 334$339,2015$.

[19] K. Remya, C. Sangeetha, and C. Suriyakala, "Cross layer Design of energy efficient protocol using adjustable sleeping windows in MANETS", In: Proc. of Global Conference on Communication Technologies, pp. 520-524, 2015.

[20] D. Khan, P. Ball, and G. Childs, "Routing in wireless ad hoc networks jointly optimized for minimum transmission power and maximum network lifetime", In: Proc. of the 8th IEEE, IET International Symposium on Communication Systems, Networks and Digital Signal Processing, pp. 56-65, 2012.

[21] B. Karaoglu and W. Heinzelman, "Cooperative Load Balancing and Dynamic Channel Allocation for Cluster-Based Mobile Ad Hoc Networks", IEEE Transactions on Mobile Computing, Vol. 14, No. 5, pp. 951-963, 2015

[22] S. Shivashankar, H. Narayanagowda, G. Varaprasad, and G. Jayanthi, "Designing Energy Routing Protocol With Power Consumption Optimization in MANET", IEEE Transactions on Emerging Topics In Computing, Vol. 2, No. 2, pp. 192-197, 2014

[23] R. Nair and S. IndiraGandhi, "Performance Analysis of Threshold Based Routing in Mobile Ad Hoc Networks", In: Proc. of the 3rd International Conference on Signal Processing, Communication, and Networking, pp.89-94, 2015.
[24] G. Walikar and R. Biradar, "Reliable and Energy Efficient Hybrid Multicast Routing in Mobile Ad Hoc Networks", In: Proc. of International Conference on Signal Processing, Communication, Power and Embedded System, pp. 27-36, 2016.

[25] M. Yoshimachi and Y. Manabe, "Battery Power Management Routing Considering Participation Duration for Mobile Ad Hoc Networks", Journal of Advances in Computer Networks, Vol. 4, No. 1, pp. 13-18, 2016.

[26] S. Nallusamy, S. Appavupillai, and S. Ponnusamy, "Mobile Agents based Reliable and Energy Efficient Routing Protocol for MANET", International Journal of Intelligent Engineering and Systems, Vol. 9, No. 3, pp. 110-116, 2016.

[27] N. Boddu, R. Vatambeti, and V. Bobba, "Achieving Energy Efficiency and Increasing the Network Life Time in MANET through Fault Tolerant Multi-Path Routing", International Journal of Intelligent Engineering and Systems, Vol. 10, No. 3, pp. 166-172, 2017.

[28] S. Ghode and K. Bhoyar, " NEMA: Node Energy Monitoring Algorithm for Zone Head Selection in Mobile Ad-Hoc Network using Residual Battery Power of Node", In: Proc. of the IEEE International Conference on Wireless Communications, Signal Processing and Networking, pp. 2054-2059, 2016. 